\title{
Rezidivierende multifokale Osteomyelitis ohne Dermatose
}

\section{Recurrent multifocal osteomyelitis in the absence of skin disease}

Autoren

Institut

\section{$\begin{array}{lll}\text { S. Lamparter } & \text { E. List-Hellwig } & \\ & \text { O. Basten }\end{array}$}

${ }^{1}$ Abteilung für Innere Medizin, Diakonie-Krankenhaus Wehrda, Marburg

2 Abteilung für Radiologie Diakonie-Krankenhaus Wehrda, Marburg

3 Institut für Pathologie und Zytologie, Marburg
Osteologie, Dermatologie

Schlüsselwörter

SAPHO-Syndrom

chronisch rekurrierende multifokale Osteomyelitis

Orphan disease

OHyperostose

\section{Keywords}

DAPHO syndrome

chronic recurrent multifocal osteomyelitis

hyperostosis eingereicht 06.11 .2013

akzeptiert $\quad 02.04 .2013$

Bibliografie

DOI $10.1055 / \mathrm{s}-0033-1343124$

Dtsch Med Wochenschr 2013;

138: 886-890 - (c) Georg Thieme Verlag KG · Stuttgart - New York . ISSN 0012-0472

\section{Korrespondenz}

\section{Dr. Steffen Lamparter}

Diakonie-Krankenhaus Wehrda, Abteilung für Innere Medizin Hebronberg 5

35041 Marburg

Tel. 06421/808-0

Fax 06421/808-567

eMail Dr.S.Lamparter@

dkh-wehrda.de

\section{Zusammenfassung \\ $\nabla$}

Anamnese und klinischer Befund: Ein 64-jähriger Patient stellte sich mit Schmerzen und einer symmetrischen Schwellung beider Klavikulae vor. Fieber und Schüttelfrost wurden auf Nachfrage verneint. Anamnestisch eruierbar waren eine Mandibula-Resektion nach Osteomyelitis vor 27 Jahren sowie rezidivierende Knochenschmerzen im Bereich des Sternums und der Brustwirbelsäule - Hautsymptome wurden verneint.

Untersuchungen: Laborchemisch fand sich bei normwertigen Leukozytenzahlen ein stark erhöhtes C-reaktives Protein und eine stark beschleunigte Blutsenkung. Die Autoimmunserologie war negativ, aerobe und anaerobe Blutkulturen blieben ohne Keimnachweis. Konventionell radiologisch fanden sich ausgedehnte fleckige und überwiegend osteosklerotische Veränderungen mit einzelnen zystischen Läsionen im Bereich beider Klavikulae, vereinbar mit einer chronischen Osteomyelitis. In der Skelettszintigraphie waren fokale Mehranreicherungen im Bereich der Klavikulae und in geringerer Ausprä-

\section{Einleitung}

Das SAPHO-Syndrom (Synovitis, Akne, Pustulosis, Hyperostosis, Osteitis) ist durch eine chronisch entzündliche Knochenerkrankung und durch Hautsymptome gekennzeichnet und wird auch als „Skibo“-Syndrom (Skin-bone disease) des Erwachsenen bezeichnet $[6,11]$. Im Folgenden berichten wir über einen Patienten mit einer chronisch rezidivierenden Osteomyelitis der Mandibula, des Sternums, der Brustwirbelsäule und beider Klavikulae, bei dem die Diagnose eines SAPHO-Syndroms durch eine fehlende Hautbeteiligung und einen chronisch remittierenden Verlauf mit atypischem Beginn verzögert gestellt wurde. gung auch im Bereich der Brustwirbelsäule nachweisbar. Eine Knochenbiopsie aus der Klavikula ergab das Bild einer Osteitis bzw. chronischen Osteomyelitis mit mäßig zellreicher Markraumfibrose und positiver Antikörperreaktion des Makrophagenmarkers CD68 und des Plasmazellmarkers CD138. Die Zusammenschau der Befunde sprach für die Diagnose eines SAPHO-Syndroms (Synovitis, Akne, Pustulosis, Hyperostosis, Osteitis) mit fehlender Hautbeteiligung.

Therapie und Verlauf: Es folgte eine symptomatische Therapie mit NSAR, Bisphosphonaten und Azithromycin, worunter bereits kurzfristig eine deutliche Beschwerdebesserung erzielt werden konnte.

Folgerung: Das SAPHO-Syndrom ist ein sehr seltener Symptomkomplex, welcher durch entzündliche osteoartikuläre Beschwerden und Hautsymptome charakterisiert ist. Die Diagnose kann allerdings durch ein variables Befallsmuster und teils fehlender Hautsymptome erschwert sein. Die Therapie ist symptomorientiert und besteht aus NSAR, Bisphosphonaten und ggfs. Glukokortikoiden.

\section{Kasuistik}

\section{Anamnese}

Bei dem 64-jährigen Patienten wurde 27 Jahre zuvor eine Mandibula-Resektion nach rezidivierender Osteomyelitis notwendig. Nach Knochenspanentnahme aus dem Os ileum erfolgte zum damaligen Zeitpunkt eine Aufbauplastik des Unterkiefers. Anamnestisch wurden verschiedene Antibiotika eingesetzt, wobei histologisch eine chronisch sklerosierende Osteomyelits ohne Malignitätsverdacht vorlag. Anschließend war der Patient jahrelang beschwerdefrei, bis vor etwa 4 Jahren starke Schmerzen am Sternum auftraten. Szintigraphisch fand 
sich in diesem Bereich eine Mehranreicherung, so dass in Zusammenschau mit dem CT-Befund ohne Nachweis eines spezifischen Erregers die Diagnose einer Osteomyelitis des Sternums gestellt und ex juvantibus mit Antibiotika therapiert wurde. Erneut folgte ein weitgehend beschwerdefreies Intervall mit rezidivierend auftretenden Wirbelsäulenbeschwerden, welche als degenerativer Genese gedeutet und symptomatisch therapiert wurden. Anamnestisch waren zu keinem Zeitpunkt Hinweiszeichen auf eine Hauterkrankung, insbesondere auf eine Akne conglobata bzw. eine pustulöse Dermatitis oder Psoriasis eruierbar. Zum Aufnahmezeitpunkt klagte der Patient über stärkste Schmerzen im Bereich der Klavikulae mit symmetrischen Schwellungen. Temperaturen und Schüttelfrost wurden verneint.

\section{Körperlicher Untersuchungsbefund}

Adipöser Patient in gering reduziertem Allgemeinzustand (180 $\mathrm{cm} / 100 \mathrm{~kg}$ ). Blutdruck 145/90 mmHg, Herzfrequenz 80/min. Abdomen unauffällig. Symmetrische und stark schmerzhafte Schwellung beider Klavikulae, schmerzhafte Bewegungseinschränkung der thorakalen BWS, sonst unauffälliger Befund.

\section{Klinisch-chemische Untersuchungen}

Laborchemisch fanden sich die Leukozytenzahlen im Normbereich. Das C-reaktive Protein $(167 \mathrm{mg} / \mathrm{l}[<5 \mathrm{mg} / \mathrm{l}])$ und Fibrinogen (> 920 [180-350 mg/l]) waren stark erhöht, die BSG mit 105/109 stark beschleunigt; Ferritin (379 ng/ml [23,9-336,2 ng/ml]) war erhöht. Die alkalische Phosphatase fand sich im Normbereich (123 U/1 [38-126 U/1]). Außerdem bestand eine normochrome Anämie (10.4 g/dl [12-18 mg/dl]). Rheumafaktor, ANA, c- und p-ANCA waren negativ. Die HLA-Typisierung (HLA-A1, A30(19), B57(17), B49(21), Cw6, Cw7, Bw4) ergab keinen Hinweis auf das Vorliegen von HLA-B27 und HLA-B8, bei denen eine Assoziation mit dem SAPHO-Syndrom beschrieben wurden. Mehrere aerobe und anaerobe Blutkulturen blieben ohne Keimnachweis. In den intraoperativ während der Knochenmarkbiopsie gewonnen Kulturen waren Zeichen einer spärlichen Kontamination mit Staphylococcus capitis nachweisbar.

\section{Ergänzende Untersuchungen}

Transthorakale und transösophageale Echokardiographie Global und regionale normale linksventrikuläre Funktion bei LV-Dilatation (LVEDD $63 \mathrm{~mm}$ ). Die Aorten- und Mitralklappe waren gering sklerosiert mit regelrechter Funktion, die Trikuspidal- und Pulmonalklappe wurden unauffällig dargestellt. Es ergaben sich keine Hinweiszeichen auf flottierende Läsionen, bzw. Zeichen einer Endokarditis.

Röntgen Thorax Konventionell radiologisch fanden sich ausgedehnte fleckige und überwiegend osteosklerotische Veränderungen mit einzelnen zystischen Läsionen im Bereich beider Klavikulae. Der Befund war vereinbar mit einer chronischen Osteomyelitis.

Skelettszintigraphie Fokale Mehranreicherung im Bereich beider Klavikulae, in geringerer Ausprägung auch im Bereich der Brustwirbelsäule ( Abb.1).

CT Thorax, nativ Osteolysen und osteoplastische Reaktionen der Klavikula beidseits, vereinbar mit einer multifokalen Osteomyelitis. Destruktion der Sternoklavikulargelenke mit Ausbildung einer sternoklavikulären Hyperostose. Massiv aufgetriebe-

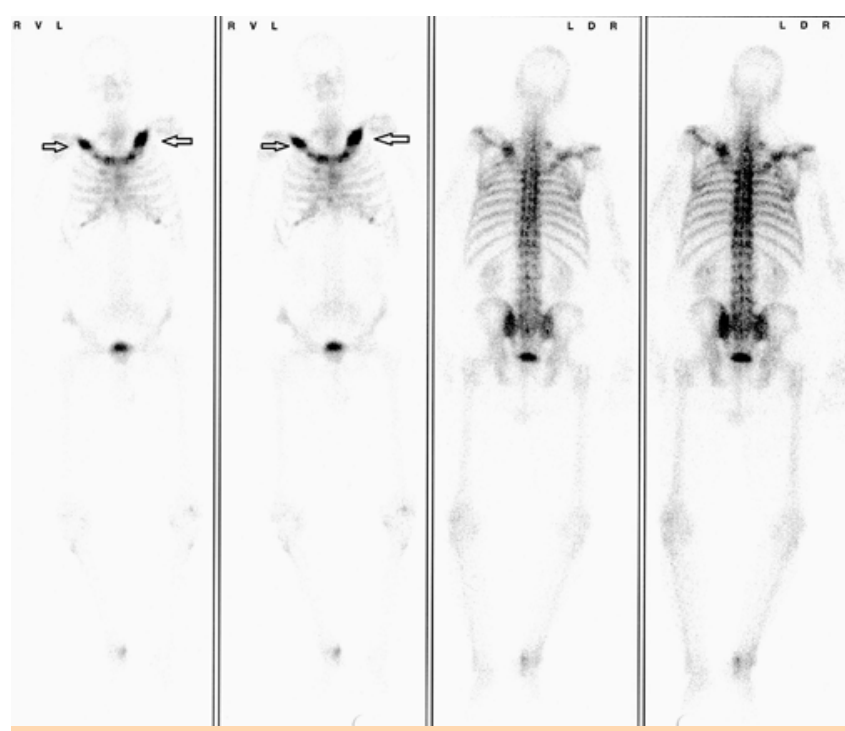

Abb.1 Skelett-Szintigraphie. Fokale Mehranreicherung im Bereich beider Klavikulae und geringer auch in der Brustwirbelsäule. Kein Aktivitätsnachweis im Manubrium sterni.

nes und vermehrt sklerosiertes Sternum. Spondyloarthropathie mit überbrückenden Osteophyten im Sinne eines M. Forestier. (O Abb.2).

Histologie aus der Klavikula Bild einer Osteitis bzw. chronischen Osteomyelitis mit mäßig zellreicher Markraumfibrose (- Abb.3). Unregelmäßiger Geflechtknochen, mit wenigen Entzündungszellen. Positive Antikörperreaktion des Makrophagenmarkers CD68 und des Plasmazellmarkers CD138.

\section{Therapie und Verlauf}

Der Patient wurde wegen stärkster Schmerzen zunächst mit Oxycodon $(10 \mathrm{mg} / \mathrm{d})$ in Kombination mit Dicofenac $(150 \mathrm{mg} / \mathrm{d})$ behandelt. Darunter besserte sich das Beschwerdebild bereits deutlich. Darüber hinaus leiteten wir eine Therapie mit Azithromycin für 4 Wochen ein (off-label-use). Ergänzt wurde die Therapie durch die Gabe von Bisphosphonaten (initial Pamidronat $30 \mathrm{mg}$ i.v., anschließend Alendronat $70 \mathrm{mg} /$ Woche). Im Rahmen einer Kontrolluntersuchung nach 4 Wochen war der Patient weitgehend beschwerdefrei, obwohl laborchemisch unverändert erhöhte Entzündungsparameter (C-reaktives Protein 95 mg/l) und eine beschleunigte BSG (94/99) vorhanden war.

\section{Diskussion}

Das SAPHO-Syndrom beschreibt einen sehr seltenen, heterogenen Symptomkomplex und wird zu den sogenannten „Orphan diseases“ gezählt (www.orpha.net) [11,12]. Die genaue Prävalenz ist unbekannt. Nosologisch ist es eng verwandt mit der chronisch rekurrierenden multifokalen Osteomyelitis (CRMO) [4] und dem Komplex der seronegativen Spondylarthropathien. Eine Abgrenzung der einzelnen Krankheitsentitäten ist teils erschwert und in der Literatur uneinheitlich. So gilt die CRMO als Variante eines SAPHO-Syndroms des Kindesalters und ist charakterisiert durch eine häufig multifokale, gelegentlich aber auch solitär entzündliche Knochenerkrankung mit lokalen Schmerzen und Schwellungen bei vorwiegender Beteiligung der Metaphysen der Röhrenknochen [9]. Die CRMO tritt im Gegensatz zum SAPHO-Syndrom 

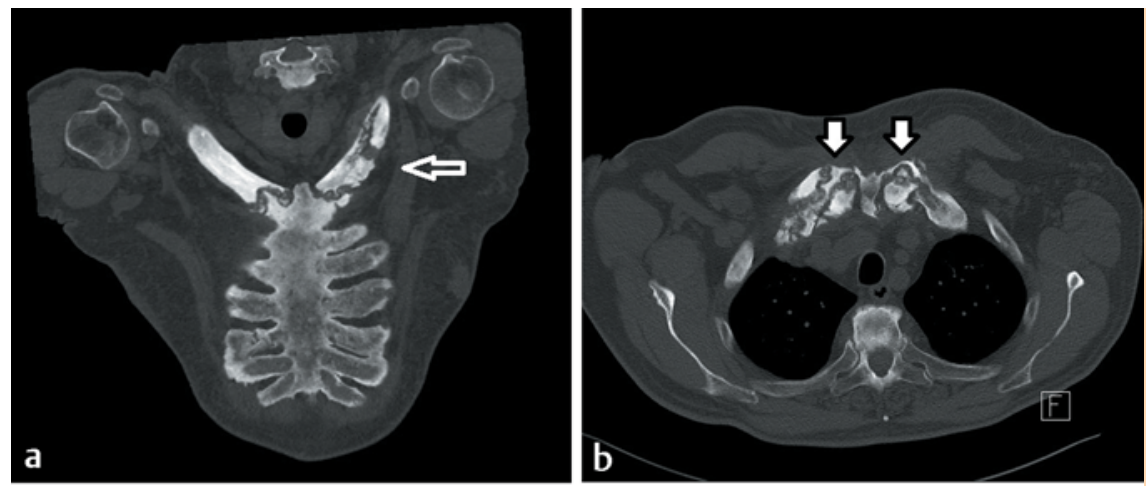

Abb.2 Computertomographie des Thorax. a) Darstellung des Sternums in multiplaner Rekonstruktionstechnik. Beidseits zeigen sich durchgehende Verknöcherungen der Sternokostalgelenke und Nachweis von bizarr geformten Osteolysen in den Klavikulae. b) Kolbige Auftreibung des Kostoklavikulargelenks mit teils osteoplastisch, teils osteolytischer Reaktion. c) Thorax seitlich, erhebliche Verdickung des Manubrium sterni und entzündlichen Veränderungen mit Kyphosierung der Brustwirbelsäule. d) 3-D-Rekonstruktion des knöchernen Thorax.
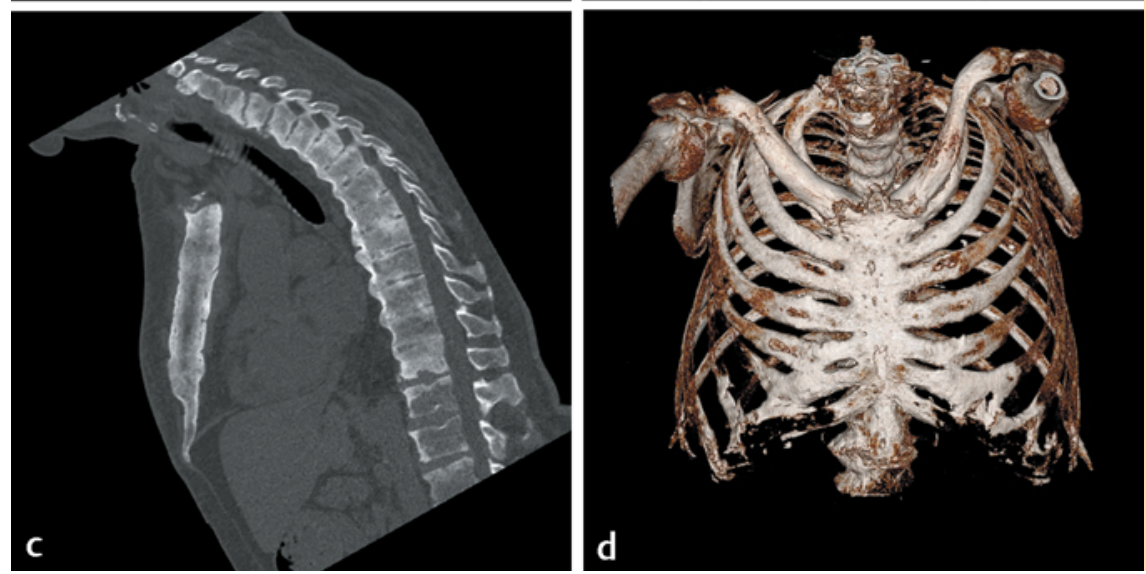

überwiegend bei Mädchen im Kindesalter auf und ist begleitet von subfebrilen Temperaturen und einem allgemeinen Krankheitsgefühl. Wie beim SAPHO-Syndrom findet sich laborchemisch bei der CRMO eine unspezifische BSG- und CRP-Erhöhung. Der Verlauf ist - ähnlich wie beim SAPHO-Syndrom - häufig chronisch mit Phasen der Remission und Exazerbation [5]. Die Prävalenz der CRMO wurde in der Literatur mit 0,04\% angegeben [11]. Darüber hinaus findet sich beim SAPHO-Syndrom in Abgrenzung zu den seronegativen Spondylarthropathien mit meist symmetrisch lokalisierter Sakroileitis eine überwiegend unilaterale Beteiligung der Sakroilikal-Gelenke mit prädominanter Hyperostose und Sklerose des Os ileum [10]. Obwohl keine klinisch validierten Diagnosekriterien für das klinisch heterogene Bild des SAPHOSyndroms existieren, wird in der Literatur häufig auf die von Benhamou et al. [3] etablierten Kriterien verwiesen. Diagnostische Kriterien für das Vorliegen eines SAPHO-Syndroms sind dabei

1. eine osteoartikuläre Manifestation der Akne conglobata, Akne fulminans oder der Hidradenitis suppurativa,

2. eine osteoartikuläre Manifestation einer palmoplantaren Pustulosis,

3. eine Hyperostosis v.a. der vorderen Thoraxwand mit oder ohne Dermatose und

4. eine CRMO des axialen oder peripheren Skelettsystems mit oder ohne Dermatose.

Die Erstdiagnose eines SAPHO-Syndroms erfolgt meist im mittleren Lebensalter. Im Vordergrund der klinischen Symptomatik steht dabei eine ossäre Beteiligung der vorderen Thoraxwand in etwa 60-70\% der Fälle, gefolgt von einer Beteiligung der Brustwirbelsäule. Hautsymptome können, wie in unserem Fall beschrieben, fehlen bzw. erst nach Jahrzehnten auftreten. Eine Beteiligung der Mandibula, wie initial in unserem Fall, tritt beim SAPHO-Syndrom in lediglich etwa 10\% der Fälle auf [15]. In etwa 3 - 7,5\% der Fälle kann es zu einer chronisch entzündlichen Darmerkrankung kommen, wobei der Morbus Crohn häufiger als die Colitis ulcerosa ist [9]. Die Ätiologie der Erkrankung ist weitgehend unbekannt, diskutiert wird aber eine mögliche Triggerung durch Propionibacterium acnes [7]. Außerdem werden immunologische und hereditäre Faktoren bei der Pathogenese des SAPHO-Syndroms diskutiert [9]. Autoantikörper sind - wie bei unserem Patienten - in der Regel nicht nachweisbar. Die Erkrankung verläuft häufig chronisch progredient mit Phasen der Remission. Wie in unserem Fall beschrieben, ist das SAPHO-Syndrom durch osteoartikuläre Schmerzen mit Betonung der Sternoklavikulargelenke charakterisiert. Zum Aufnahmezeitpunkt sind in Übereinstimmung mit dem klinischen und szintigraphischen Befund die Klavikuae beidseits entzündlich verändert. Obwohl multifokal chronische bzw. postentzündliche Veränderungen der Brustwirbelsäule und des Sternums im CT dokumentiert werden konnten, fand sich szintigraphisch kein Anhalt für eine aktive entzündliche Veränderung in diesen Regionen. Da bei dem Patienten keine mit dem SAPHO-Syndrom assoziierte Hauterkrankung vorhanden bzw. anamnestisch eruierbar war, erfolgte bislang keine Zuordnung zum SAPHO-Syndrom. In Zusammenschau der Befunde spricht allerdings der klinische Verlauf zusammen mit dem histologischen Befund für diese Diagnose.

Im Vordergrund der Diagnostik steht die Anamnese und der klinisch rheumatologische Befund. Laborchemisch finden sich erhöhte Entzündungsparameter mit beschleunigter BSG und erhöhtem C-reaktiven Protein. Spezifische Laborparameter die ein SAPHO-Syndrom charakterisieren, bestehen nicht. Obwohl eine Assoziation des SAPHO-Syndroms mit HLA-B27 und -B8 beschrieben wurde, ist die Bestimmung des HLA-Status nicht diagnostisch verwertbar [9, 14]. Im Vordergrund der apparativen Diagnostik stehen die konventionelle Röntgendiagnostik, die Knochenszintigraphie und das MRT. Die ossären Veränderungen des SAPHO-Syndroms gelten nicht als Präkanzerose. Eine Abgrenzung zu malignen oder infektiösen Prozessen allein auf dem Bo- 

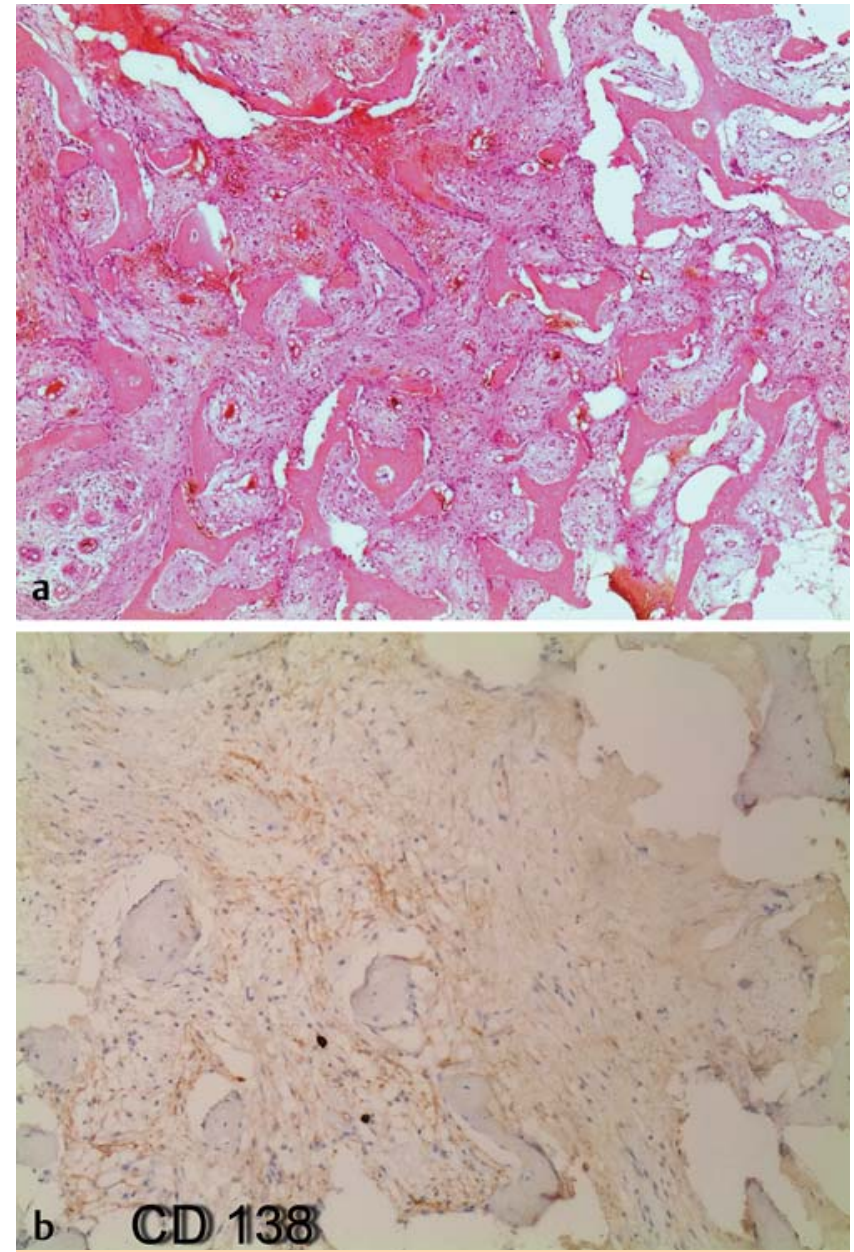

Abb.3 Bild einer Osteitis bzw. chronischen Osteomyelitis mit mäßig zahlreicher Markraumfibrose. a) Unregelmäßiger Geflechtknochen mit vermehrter Umbaureaktion bei chronischer Osteitis und Osteomyelitis mit wenigen gemischten Entzündungszellen und mäßig zellreicher Markraumfibrose (HE- Färbung, Vergrößerung 40x). b) CD138-Antikörperreaktion mit Darstellung von wenigen Plasmazellen (Vergrößerung 100x).

den der Bildgebung ist allerdings gelegentlich erschwert und muss ggfs. histologisch erfolgen. Die Prognose der Patienten mit SAPHO-Syndrom ist quoad vitam günstig, so dass die Therapie symptomorientiert und nach der individuellen Schmerzsymptomatik des Patienten ausgerichtet ist. Bei chronischen Verläufen kann es allerdings in Einzelfällen zu einer körperlichen Behinderung mit Bewegungseinschränkung kommen. Darüber hinaus sind Einzelfälle von Thrombosen der V. subclavia und der V. cava superior durch eine mechanische Gefäßkompression beschrieben [16] .

Aufgrund der Seltenheit der Erkrankung existieren keine systematischen Therapiestudien, die einen spezifischen therapeutischen Ansatz zur Beeinflussung des Krankheitsverlaufs dokumentieren. Einzelne Fallberichte deuten auf einen günstigen Effekt einer lokalen und systemischen Glukokortikoid-Therapie. Außerdem wird die Gabe von Bisphosphonaten und NSAR bei Knochenschmerzen empfohlen [8]. Basierend auf einzelnen Kasuistiken mit Therapierfolgen durch Azithromycin [1] erfolgte bei unserem Patienten trotz fehlendem Erregernachweis eine Antibiotika-Therapie über 4 Wochen. Wie von Schilling und Wagner [13] beschrieben, werden neben einem antibakteriellen Effekt auch immunmodulatorische und antiphlogistische Wir- kungen durch Azithromycin vermutet. Ob die von dem Patienten berichtete Beschwerdebesserung auf den antibakteriellen Effekt, auf die antiphlogistische Wirkung der NSAR oder aber auf die Kombination mit Bisphosphonaten zurückzuführen ist, lässt sich nicht genau eruieren. Eine immunsuppressive bzw. immunmodulatorische Therapie durch systemische Glukokortikoide, Methotrexat und Leflunomid kann bei progredienten Verläufen mit intraktablen Schmerzen erwogen werden. Einzelne Berichte deuten darüber hinaus auf einen positiven Effekt von TNF-alpha-Rezeptorantagonisten zur Therapie bei refraktären Verläufen [2]. Im vorliegenden Fall wurde bei weitgehender Beschwerdefreiheit unter einer symptomatischen Therapie keine immunsuppressive Therapie initiiert.

Zusammenfassend ist die Diagnose des SAPHO-Syndroms durch das variable klinische Erscheinungsbild häufig erschwert und die Diagnosestellung kann - wie in unserem Fall beschrieben - durch das Fehlen typischer Hautveränderungen verzögert sein. Im Vordergrund der Diagnostik stehen neben der Anamnese die klinische Präsentation, die Bildgebung einschließlich Skelettszintigraphie und ggfs. eine histologische Sicherung. Die Therapie ist symptomorientiert und bei progredientem Verlauf evtl. durch immunsuppressive bzw. immunmodulatorische Substanzen zu ergänzen.

\section{Konsequenz für Klinik und Praxis:}

- Das SAPHO-Syndrom ist eine sehr seltene Erkrankung und ist charakterisiert durch den Symptomkomplex Synovitis, Akne, Pustulosis, Hyperostosis und Osteitis. Nicht alle Symptome müssen gleichzeitig vorliegen, um die Diagnose zu etablieren. Insbesondere kann die Hautbeteiligung den Knochenveränderungen vorausgehen, oder aber auch mit einer Latenz von Jahrzehnten folgen.

- Die Diagnose ist bei atypischem Verlauf erschwert. Eine Abgrenzung zur CRMO und zur seronegativen Spondylarthropathie ist im Einzelfall schwierig.

- Diagnostische Laborparameter existieren nicht

- Die Differenzialdiagnose zu malignen und infektiösen Knochenerkrankungen ist alleine aufgrund der konventionellen Bildgebung häufig erschwert.

- Eine frühe Diagnose ist wichtig, um unnötige invasive diagnostische Prozeduren zu vermeiden und um eine symptomorientierte Therapie mit NSAR, Bisphosphonaten und einer lokalen und ggfs. systemischen Glukokortikoid-Gabe rechtzeitig einzuleiten.

- Bei einem therapierefraktären Verlauf sind immunmodulatorische und immunsuppressive Therapieoptionen zu erwägen.

Widmung: Diese Arbeit ist Herrn Dr. med. Walter Gleichmann, Chefarzt der Abteilung für Innere Medizin und Geriatrie - Diakonie-Krankenhaus Marburg Wehrda, zum 65. Geburtstag gewidmet.

Autorenerklärung: Die Autoren erklären, dass sie keine finanzielle Verbindung mit einer Firma haben, deren Produkt in diesem Beitrag eine Rolle spielt (oder mit einer Firma, die ein Konkurrenzprodukt vertreibt). 


\section{Abstract}

\section{Recurrent multifocal osteomyelitis in the absence of skin disease}

History and admission findings: A 64-year-old man complained of pain and a symmetric swelling of the clavicles. There were no fever and chills. 27 years earlier, a resection of the mandible was necessary, due to osteomyelitis. In the past, he complained of recurrent episodes of pain in the sternum and in the thoracic spine. Skin disorders were not reported.

Investigations: The leucocyte count was within the normal range, however, levels of c-reactive protein and the erythrocyte sedimentation rate were increased. Autoantibodies and blood cultures were negative. On X-ray, osteosclerotic and cystic lesions in both clavicles were found, consistent with osteomyelitis. A bone scintigraphy revealed increased radionuclide activity in the clavicles and the sternum. A bone biopsy sample from the clavicle revealed signs of osteitis with fibrosis together with $C D$ 68 und CD 138 positive cells. These findings indicated the diagnosis of a SAPHO syndrome (Synovitis, Acne, Pustulosis, Hyperostosis, Osteitis) without skin disease.

Treatment and course: NSAIDs, bisphosphonates and azithromycin were administered, thereby sufficient pain relief was rapidly achieved.

Conclusion: SAPHO syndrome is a rare disease complex including osteoarticular disorders which are frequently accompanied by skin disease. However, the diagnosis may be difficult due to a variable clinical manifestation and especially - like in our case if skin disease is absent. Current treatment options are not evidence-based due to the rarity of the syndrome but include NSAIDs, bisphosphonates and glucocorticoids.

\section{Glossar}

SAPHO = Synovitis, Akne, Pustulosis, Hyperostosis, Osteitis

$\mathrm{CRMO}=$ Chronisch rekurrierende multifokale Osteomyelitis

NSAR $=$ Nicht-steroidale Antirheumatika

MRT $=\quad$ Magnetresonanztomographie
Literatur

1 Assmann G, Kueck O, Kirchhoff $T$ et al. Efficacy of antibiotic therapy for SAPHO syndrome is lost after its discontinuation: an interventional study. Arthritis Res Ther 2009; 11: R140

2 Ben Abdelghani K, Dran DG, Gottenberg JE et al. Tumor necrosis factor-alpha blockers in SAPHO syndrome. J Rheumatol 2010; 37: 1699-1704

3 Benhamou CL, Chamot AM, Kahn MF. Synovitis-acne-pustulosis hyperostosis-osteomyelitis syndrome (SAPHO). A new syndrome among the spondyloarthropathies?. Clin Exp Rheumatol 1988; 6: 109-112

4 Bjorksten B, Gustavson KH, Eriksson B et al. Chronic recurrent multifocal osteomyelitis and pustulosis palmoplantaris. J Pediatr 1978; 93: 227-231

5 Catalano-Pons C, Comte A, Wipff J et al. Clinical outcome in children with chronic recurrent multifocal osteomyelitis. Rheumatology 2008; 47: 1397-1399

6 Chamot AM, Benhamou CL, Kahn MF et al. [Acne-pustulosis-hyperostosis-osteitis syndrome. Results of a national survey. 85 cases]. Rev Rhum Mal Osteoartic 1987; 54: 187-196

7 Hurtado-Nedelec M, Chollet-Martin S, Nicaise-Roland P et al. Characterization of the immune response in the synovitis, acne, pustulosis, hyperostosis, osteitis (SAPHO) syndrome. Rheumatology 2008; 47: 1160-1167

8 Marshall H, Bromilow J, Thomas AL et al. Pamidronate: a novel treatment for the SAPHO syndrome? Rheumatology 2002; 41: 231-233

9 Nguyen MT, Borchers A, Selmi C et al. The SAPHO syndrome. Sem Arthritis Rheum 2012; 42: 254-265

10 Salles $M$, Olive A, Perez-Andres $R$ et al. The SAPHO syndrome: a clinical and imaging study. Clin Rheumatol 2011; 30: 245-249

11 Schilling F. SAPHO-SyndromOrphanet, Orpha-Kennnummer 793, 2006; www.orpha.net/data/patho/DE/de-sapho.pdf

12 Schilling F, Kessler S. [SAPHO syndrome: clinico-rheumatologic and radiologic differentiation and classification of a patient sample of 86 cases]. Z Rheumatol 2000; 59: 1-28

13 Schilling $F$, Wagner $A D$. [Azithromycin: an anti-inflammatory effect in chronic recurrent multifocal osteomyelitis? A preliminary report]. Z Rheumatol 2000; 59: 352-353

14 Toussirot E, Dupond JL, Wendling D. Spondylodiscitis in SAPHO syndrome. A series of eight cases. Ann Rheum Dis 1997; 56: 52-58

15 Zemann $W$, Pau $M$, Feichtinger $M$ et al. SAPHO syndrome with affection of the mandible: diagnosis, treatment, and review of literature. Oral Surg Oral Med Pahtol Oral Radiol Endod 2011; 111: 190-195

16 Kawabata T, Morita Y, Nakatsuka A et al. Multiple venous thrombosis in SAPHO syndrome. Ann Rheum Dis 2005; 64: 505-506 